\title{
Dynamic anti-plane behavior for interacting cracks emanating from the edges of the circular cavities in piezoelectric bimaterials
}

\author{
Lixia $\mathrm{Wu}^{1, \mathrm{a}}$, Dong $\mathrm{Li}^{1, \mathrm{~b}^{*}}$ and Yanru Ren ${ }^{1, \mathrm{c}}$ \\ ${ }^{1}$ Department of Civil Engineering, Hebei Jiaotong Vocational \& Technical College, Shijiazhuang \\ 050091, PR China \\ addwlx@126.com, ${ }^{\mathrm{b}}$ lidong242@163.com, ${ }^{\mathrm{c}}$ rener112@163.com
}

Keywords: circular cavity, radial crack, Green's function, crack-division, dynamic stress intensity factor (DSIF), piezoelectric bimaterials

Abstract. In this paper, we examine the dynamic anti-plane behavior of interacting redial cracks emanating from the edges of two interfacial circular cavities in piezoelectric bimaterials under incident anti-plane shearing wave (SH-wave). Green's functions for a semi-infinite piezoelectric medium with two circular cavities are determined by using complex variable method. The present problem is reduced to a series of Fredholm integral equations of the first kind based on Conjunction and crack-division techniques. Typical examples are provided to show the effect of the piezoelectric constants, structural geometry, and the wave frequencies of incident wave upon the local stress field around the crack tips.

\section{Introduction}

Piezoelectric materials are widely used in the fields of aeronautics, marine, automobile as sensors, actuators, etc. Occasionally, fracture or failure often acquire in piezoelectric devices during the manufacturing, polling process because of the material's brittle nature. Therefore, researchers paid great attention to the failure behaviors and influences caused by defects in piezoelectric materials.

In recent years, Narita and Shindo [1] studied the scattering of horizontally polarized shear waves by a finite crack in the piezoelectric material. Wang [2] examined the dynamic behavior of interacting interfacial cracks between two piezoelectric media under anti-plane mechanical and in-plane electric loading. Zhong and $\mathrm{Li}$ [3] gave a closed-form solution for two collinear cracks in a piezoelectric strip. Zhou and Wang [4] obtained the basic solution of two parallel non-symmetric permeable cracks in piezoelectric materials. We can see that these documents are focus on the crack defects. But in fact, there exist various complicated defects during manufacture and service of the holed piezoelectric structures, e.g., cracks emanating from the circular cavities. Some work has paid attention to this complicated defects, such as Wang and Gao [5], Guo et al. [6]. They studied this complicated defects at the static situation and their conclusions indicate that the simplification of an interfacial radical crack on the cavity to a straight-line interfacial crack may not be constantly partial to safety. It should be mentioned, however, that piezoelectric materials are mostly being used in situation where dynamic loading is involved, such as the vibration control of smart structures under impact loading.

The objective of the present paper is to provide a theoretical treatment of the dynamic interaction between two interfacial radial cracks in piezoelectric bimaterials under the incident anti-plane shearing wave. A permeable crack model is employed in the paper. The theoretical formulations are constructed by the Green's function, Conjunction and crack-division techniques.

\section{Problem statement and basic equation}

The analysis model is that two radial cracks emanating from the edge of two interfacial circular cavities in piezoelectric bimaterials, as shown in Fig. 1. A global Cartesian coordinate xoy and two local systems $x_{j} o_{j} y_{j}(j=1,2)$ are employed to characterize the different radial cracks. The poling direction is the positive $z$-axis. $A_{1}, A_{2}, d, R_{1}$ and $R_{2}$ are represent the cracks' lengths, the distance between the crack's tips and the radii of the circular cavities. The model is subjected to a harmonic incident wave of frequency $\omega$ with an incident angle $\alpha_{0}$. 


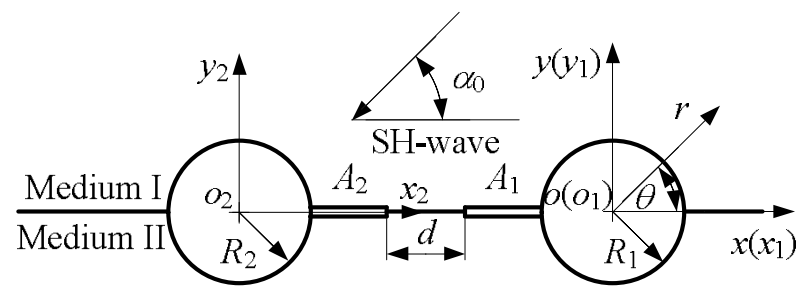

Fig. 1 Piezoelectric bimaterials with radial cracks emanating from the edges of circular cavities The boundary conditions of the cracks and the circular cavity $j$ can be expressed respectively as

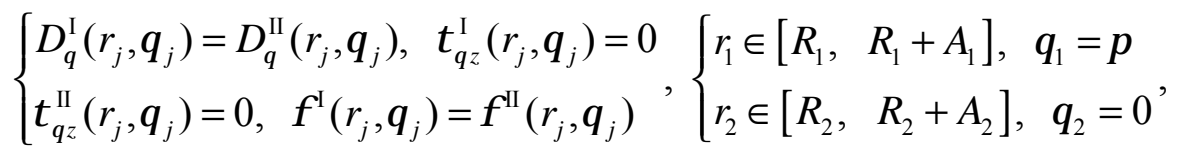

1)

$$
\left\{\begin{array}{l}
\tau_{r z}^{\mathrm{I}}\left(r_{j}, \theta_{j}\right)=0, \quad \tau_{r z}^{\mathrm{II}}\left(r_{j}, \theta_{j}\right)=0 \\
\phi^{\mathrm{I}}\left(r_{j}, \theta_{j}\right)=\phi^{c j}, \quad \phi^{\mathrm{II}}\left(r_{j}, \theta_{j}\right)=\phi^{c j} \quad,\left|r_{j}^{\mathrm{r}}\right|=R_{j}, \\
D_{r}^{\mathrm{I}}\left(r_{j}, \theta_{j}\right)=D_{r}^{c j}, \quad D_{r}^{\mathrm{II}}\left(r_{j}, \theta_{j}\right)=D_{r}^{c j}
\end{array}\right.
$$

2)

where $\tau_{r z}, \tau_{\theta z}, D_{r}$ and $D_{\theta}$ are two shear stress components and two electric displacements, while $\phi$ is the electric potential, respectively. The superscripts I, II and $c$ express variables in the medium I, the medium II and the circular cavity, respectively.

The governing equations of linear piezoelectricity can be specified as

$$
c_{44} \nabla^{2} w+e_{15} \nabla^{2} \phi+\rho \omega^{2} w=0, e_{15} \nabla^{2} w-\kappa_{11} \nabla^{2} \phi=0,
$$

in which, $c_{44}, e_{15}$ and $\kappa_{11}$ are shear elastic modulus, piezoelectric constant and dielectric constant of the medium, respectively; $w$ and $\rho$ are anti-plane displacement and mass density, respectively.

Introducing a complex variable $\eta=x+i y=r e^{i \theta}$ and $\bar{\eta}=x-i y=r e^{-i \theta}$, Eq. 3 can be rewritten as

$$
\frac{\partial^{2} w}{\partial \eta \partial \bar{\eta}}+\frac{1}{4} k^{2} w=0, \frac{\partial^{2} \varphi}{\partial \eta \partial \bar{\eta}}=0, \phi=\frac{e_{15}}{\kappa_{11}} w+\varphi,
$$

where $k^{2}=\rho \omega^{2} / c^{*}, c^{*}=c_{44}(1+\lambda), \lambda=e_{15}^{2} / c_{44} \kappa_{11}$.

The constitutive relations in $(\eta, \bar{\eta})$ plane for the piezoelectric material can be expressed as

$$
\begin{aligned}
& \tau_{r z}=c_{44}\left(\frac{\partial w}{\partial \eta} e^{i \theta}+\frac{\partial w}{\partial \bar{\eta}} e^{-i \theta}\right)+e_{15}\left(\frac{\partial \phi}{\partial \eta} e^{i \theta}+\frac{\partial \phi}{\partial \bar{\eta}} e^{-i \theta}\right), \\
& \tau_{\theta z}=i c_{44}\left(\frac{\partial w}{\partial \eta} e^{i \theta}-\frac{\partial w}{\partial \bar{\eta}} e^{-i \theta}\right)+i e_{15}\left(\frac{\partial \phi}{\partial \eta} e^{i \theta}-\frac{\partial \phi}{\partial \bar{\eta}} e^{-i \theta}\right), \\
& D_{r}=e_{15}\left(\frac{\partial w}{\partial \eta} e^{i \theta}+\frac{\partial w}{\partial \bar{\eta}} e^{-i \theta}\right)-\kappa_{11}\left(\frac{\partial \phi}{\partial \eta} e^{i \theta}+\frac{\partial \phi}{\partial \bar{\eta}} e^{-i \theta}\right), \\
& D_{\theta}=i e_{15}\left(\frac{\partial w}{\partial \eta} e^{i \theta}-\frac{\partial w}{\partial \bar{\eta}} e^{-i \theta}\right)-i \kappa_{11}\left(\frac{\partial \phi}{\partial \eta} e^{i \theta}-\frac{\partial \phi}{\partial \bar{\eta}} e^{-i \theta}\right) .
\end{aligned}
$$

\section{Boundary value problem and DSIFs}

Firstly, the Green's functions of elastic displacement $G_{w}$ and electric potential $G_{\phi}$, which are the fundamental solutions for a semi-infinite piezoelectric material with two circular notches impacted by 
an out-plane harmonic line source loading $\delta\left(\eta-\eta_{0}\right)$ at the horizontal surface, can be described as

$$
\begin{aligned}
& G_{w}=\frac{i}{2 c^{*}} H_{0}^{(1)}\left(k\left|\eta-\eta_{0}\right|\right)+\sum_{j=1}^{2} \sum_{m=-\infty}^{\infty} A_{m}^{j} H_{m}^{(1)}\left(k\left|\eta_{j}\right|\right) \cdot\left[\left(\frac{\eta_{j}}{\left|\eta_{j}\right|}\right)^{m}+\left(\frac{\eta_{j}}{\left|\eta_{j}\right|}\right)^{-m}\right], \\
& G_{\phi}=\frac{e_{15}}{\kappa_{11}} G_{w}+\sum_{j=1}^{2} \sum_{m=1}^{\infty}\left[B_{m}^{j} \eta_{j}^{-m}+C_{m}^{j} \bar{\eta}_{j}^{-m}\right] .
\end{aligned}
$$

The electric potential in the circular cavities can be written as

$$
G_{\phi}^{c j}=D_{0}+\sum_{m=1}^{\infty}\left[D_{m}^{j} \eta_{j}^{m}+E_{m}^{j} \bar{\eta}_{j}^{m}\right]
$$

The unknown constants $A_{m}^{j}, B_{m}^{j}, C_{m}^{j}, D_{m}^{j}$ and $E_{m}^{j}$ can be solved by Eq. 2.

Secondly, consider the piezoelectric bimaterials with two interfacial circular cavities subjected to a harmonic SH-wave directed at an angle $\square_{0}$ with the interface in medium I. The dynamic electro-elastic fields and the corresponding scattering fields which caused by two circular cavities can be given as follows:

1. Incident and scattering electro-elastic fields in medium I:

$$
\begin{aligned}
& w^{(i)}=w_{0} \cdot \exp \left[-i \frac{k_{\mathrm{I}}}{2}\left(\eta e^{-i \alpha_{0}}+\bar{\eta} e^{i \alpha_{0}}\right)\right], \phi^{(i)}=\phi_{0} \cdot \exp \left[-i \frac{k_{\mathrm{I}}}{2}\left(\eta e^{-i \alpha_{0}}+\bar{\eta} e^{i \alpha_{0}}\right)\right], \\
& w^{(i s)}=\sum_{j=1}^{2} \sum_{m=-\infty}^{\infty}{ }^{j} A_{m}^{(i s)} H_{m}^{(1)}\left(k_{\mathrm{I}}\left|\eta_{j}\right|\right)\left(\frac{\eta_{j}}{\eta_{j} \mid}\right)^{m}, \phi^{(i s)}=\frac{e_{15}^{\mathrm{I}}}{\kappa_{11}^{\mathrm{I}}} w^{(i s)}+\sum_{j=1}^{2} \sum_{m=1}^{+\infty}{ }^{j} B_{m}^{(i s)} \eta_{j}^{-m}+\sum_{j=1}^{2} \sum_{m=1}^{+\infty}{ }^{j} C_{m}^{(i s)} \bar{\eta}_{j}^{-m} .
\end{aligned}
$$

2. Reflecting and scattering electro-elastic fields in medium I:

$$
\begin{aligned}
& w^{(r)}=w_{1} \cdot \exp \left[-i \frac{k_{\mathrm{I}}}{2}\left(\eta e^{i \alpha_{0}}+\bar{\eta} e^{-i \alpha_{0}}\right)\right], \phi^{(r)}=\phi_{1} \cdot \exp \left[-i \frac{k_{\mathrm{I}}}{2}\left(\eta e^{i \alpha_{0}}+\bar{\eta} e^{-i \alpha_{0}}\right)\right], \\
& w^{(r s)}=\sum_{j=1}^{2} \sum_{m=-\infty}^{\infty}{ }^{j} A_{m}^{(r s)} H_{m}^{(1)}\left(k_{\mathrm{I}}\left|\eta_{j}\right|\right)\left(\frac{\eta_{j}}{\eta_{j} \mid}\right)^{m}, \phi^{(r s)}=\frac{e_{15}^{\mathrm{I}}}{\kappa_{11}^{\mathrm{I}}} w^{(r s)}+\sum_{j=1}^{2} \sum_{m=1}^{+\infty}{ }^{j} B_{m}^{(r s)} \eta_{j}^{-m}+\sum_{j=1}^{2} \sum_{m=1}^{+\infty}{ }^{j} C_{m}^{(r s)} \bar{\eta}_{j}^{-m} .
\end{aligned}
$$

3. Transmitting and scattering electro-elastic fields in medium II:

$$
\begin{aligned}
& w^{(t)}=w_{2} \cdot \exp \left[-i \frac{k_{\mathrm{II}}}{2}\left(\eta e^{-i \alpha_{2}}+\bar{\eta} e^{i \alpha_{2}}\right)\right], \phi^{(t)}=\phi_{2} \cdot \exp \left[-i \frac{k_{\mathrm{II}}}{2}\left(\eta e^{-i \alpha_{2}}+\bar{\eta} e^{i \alpha_{2}}\right)\right], \\
& w^{(t s)}=\sum_{j=1}^{2} \sum_{m=-\infty}^{\infty}{ }^{j} A_{m}^{(t s)} H_{m}^{(1)}\left(k_{\mathrm{II}}\left|\eta_{j}\right|\right)\left(\frac{\eta_{j}}{\left|\eta_{j}\right|}\right)^{m}, \phi^{(t s)}=\frac{e_{15}^{\mathrm{II}}}{\kappa_{11}^{\mathrm{II}}} w^{(t s)}+\sum_{j=1}^{2} \sum_{m=1}^{+\infty}{ }^{+\infty} B_{m}^{(t s)} \eta_{j}^{-m}+\sum_{j=1}^{2} \sum_{m=1}^{+\infty}{ }^{j} C_{m}^{(t s)} \bar{\eta}_{j}^{-m} .
\end{aligned}
$$

The relations of $\alpha_{0}$ and $\alpha_{2}, k_{\mathrm{I}}$ and $k_{\mathrm{II}}, w_{0}$ and $w_{1}, w_{2}, \phi_{0}$ and $\phi_{1}, \phi_{2}$ can refer to document[2].

Summation of the total electro-elastic fields are

$$
w^{\mathrm{I}}=w^{(i)}+w^{(i s)}+w^{(r)}+w^{(r s)}, \phi^{\mathrm{I}}=\phi^{(i)}+\phi^{(i s)}+\phi^{(r)}+\phi^{(r s)},
$$

and

$$
w^{\mathrm{II}}=w^{(t)}+w^{(t s)}, \phi^{\mathrm{II}}=\phi^{(t)}+\phi^{(t s)},
$$


in medium I and medium II, respectively.

Only the electric fields exist in the circular cavities, they are

$$
\begin{aligned}
& \phi_{j}^{i c}={ }^{j} D_{0}^{(i s)}+\sum_{m=1}^{\infty}\left[{ }^{j} D_{m}^{(i s)} \eta_{j}^{m}+{ }^{j} E_{m}^{(i s)} \bar{\eta}_{j}^{m}\right], \\
& \phi_{j}^{r c}={ }^{j} D_{0}^{(r s)}+\sum_{m=1}^{\infty}\left[{ }^{j} D_{m}^{(r s)} \eta_{j}^{m}+{ }^{j} E_{m}^{(r s)} \bar{\eta}_{j}^{m}\right] \\
& ) \\
& \phi_{j}^{t c}={ }^{j} D_{0}^{(t s)}+\sum_{m=1}^{\infty}\left[{ }^{j} D_{m}^{(t s)} \eta_{j}^{m}+{ }^{j} E_{m}^{(t s)} \bar{\eta}_{j}^{m}\right] \\
& )
\end{aligned}
$$

The values of the coefficients can be obtained by Eq. 2 .

Thirdly, the mathmatic model of present problem can be constructed by the conjunction and crack-division techniques, as shown in Fig. 2. The piezoelectric material is separated at $y=0$, bearing in mind the absence of the cracks. To achieve the boundary conditions at the surfaces of the cracks, a pair of negative shear stresses $-\tau_{\theta z}^{\mathrm{I}}$ and $-\tau_{\theta z}^{\mathrm{II}}$ are applied on the cracks' locations. And a pair of additional unknown shear stresses $f_{1}\left(r_{0}, \theta_{0}\right)$ and $f_{2}\left(r_{0}, \theta_{0}\right)$ are applied on the well-boned interfaces.

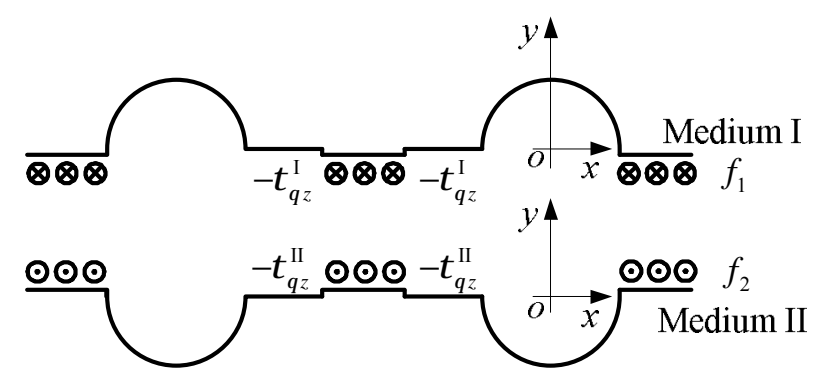

Fig. 2 Conjunction for piezoelectric bimaterials with four semi-circular notches

The continuity conditions of the shear stresses on the well-boned interfaces can be written as

$\tau_{\theta z}^{\mathrm{I}} \cos \theta_{0}+f_{1}\left(r_{0}, \theta_{0}\right)=\tau_{\theta z}^{\mathrm{II}} \cos \theta_{0}+f_{2}\left(r_{0}, \theta_{0}\right)$,

The continuity conditions of elastic displacements can be expressed as

$$
w^{\mathrm{I}}+w^{\left(f_{1}\right)}+w^{(c \mathrm{I})}=w^{\mathrm{II}}+w^{\left(f_{2}\right)}+w^{(c \mathrm{II})},
$$

in which,

$$
\begin{aligned}
w^{\left(f_{1}\right)}= & \int_{R_{2}}^{\infty} f_{1}\left(r_{02}, \pi\right) G_{w}^{\mathrm{I}}\left(r_{2}, \theta_{2} ; r_{02}, \pi\right) d r_{02}+\int_{R_{1}+A_{1}}^{R_{1}+A_{1}+d} f_{1}\left(r_{01}, \pi\right) G_{w}^{\mathrm{I}}\left(r_{1}, \theta_{1} ; r_{01}, \pi\right) d r_{01} \\
& +\int_{R_{1}}^{\infty} f_{1}\left(r_{01}, 0\right) G_{w}^{\mathrm{I}}\left(r_{1}, \theta_{1} ; r_{01}, 0\right) d r_{01} \\
w^{\left(f_{2}\right)}= & -\int_{R_{2}}^{\infty} f_{2}\left(r_{02}, \pi\right) G_{w}^{\mathrm{II}}\left(r_{2}, \theta_{2} ; r_{02}, \pi\right) d r_{02}-\int_{R_{1}+A_{1}}^{R_{1}+A_{1}+d} f_{2}\left(r_{01}, \pi\right) G_{w}^{\mathrm{II}}\left(r_{1}, \theta_{1} ; r_{01}, \pi\right) d r_{01} \\
& -\int_{R_{1}}^{\infty} f_{2}\left(r_{01}, 0\right) G_{w}^{\mathrm{II}}\left(r_{1}, \theta_{1} ; r_{01}, 0\right) d r_{01} \\
w^{(c \mathrm{I})}= & \int_{R_{1}}^{R_{1}+A_{1}} \tau_{\theta z}^{\mathrm{I}}\left(r_{01}, \pi\right) G_{w}^{\mathrm{I}}\left(r_{1}, \theta_{1} ; r_{01}, \pi\right) d r_{01}-\int_{R_{2}}^{R_{2}+A_{2}} \tau_{\theta z}^{\mathrm{I}}\left(r_{02}, 0\right) G_{w}^{\mathrm{I}}\left(r_{2}, \theta_{2} ; r_{02}, 0\right) d r_{02} \\
w^{(c \mathrm{II})}= & -\int_{R_{1}}^{R_{1}+A_{1}} \tau_{\theta z}^{\mathrm{II}}\left(r_{01}, \pi\right) G_{w}^{\mathrm{II}}\left(r_{1}, \theta_{1} ; r_{01}, \pi\right) d r_{01}+\int_{R_{2}}^{R_{2}+A_{2}} \tau_{\theta z}^{\mathrm{II}}\left(r_{02}, 0\right) G_{w}^{\mathrm{II}}\left(r_{2}, \theta_{2} ; r_{02}, 0\right) d r_{02}
\end{aligned}
$$


Substituting Eq. 15, 16 and 20 into Eq. 21, the integral equations to calculate the unknown stress $f_{1}\left(r_{0}, \theta_{0}\right)$ can be obtained as follows

$$
\begin{aligned}
& \int_{R_{2}}^{\infty} f_{1}\left(r_{02}, \pi\right)\left[G_{w}^{\mathrm{I}}\left(r_{2}, \theta_{2} ; r_{02}, \pi\right)+G_{w}^{\mathrm{II}}\left(r_{2}, \theta_{2} ; r_{02}, \pi\right)\right] d r_{02} \\
& +\int_{R_{1}+A_{1}}^{R_{1}+A_{1}+d} f_{1}\left(r_{01}, \pi\right)\left[G_{w}^{\mathrm{I}}\left(r_{1}, \theta_{1} ; r_{01}, \pi\right)+G_{w}^{\mathrm{II}}\left(r_{1}, \theta_{1} ; r_{01}, \pi\right)\right] d r_{01} \\
& +\int_{R_{1}}^{\infty} f_{1}\left(r_{01}, 0\right)\left[G_{w}^{\mathrm{I}}\left(r_{1}, \theta_{1} ; r_{01}, 0\right)+G_{w}^{\mathrm{II}}\left(r_{1}, \theta_{1} ; r_{01}, 0\right)\right] d r_{01} \\
& =w^{(t s)}(r, \theta)-w^{(i s)}(r, \theta)-w^{(r s)}(r, \theta)+\int_{R_{2}}^{\infty}\left[\tau_{\theta z}^{\mathrm{I}}\left(r_{02}, \pi\right)-\tau_{\theta z}^{\mathrm{II}}\left(r_{02}, \pi\right)\right] G_{w}^{\mathrm{II}}\left(r_{2}, \theta_{2} ; r_{02}, \pi\right) d r_{02} \quad,(\theta=0, \pi) . \\
& +\int_{R_{1}+A_{1}}^{R_{1}+A_{1}+d}\left[\tau_{\theta z}^{\mathrm{I}}\left(r_{01}, \pi\right)-\tau_{\theta z}^{\mathrm{II}}\left(r_{01}, \pi\right)\right] G_{w}^{\mathrm{II}}\left(r_{1}, \theta_{1} ; r_{01}, \pi\right) d r_{01} \\
& -\int_{R_{1}}^{\infty}\left[\tau_{\theta z}^{\mathrm{I}}\left(r_{01}, 0\right)-\tau_{\theta z}^{\mathrm{II}}\left(r_{01}, 0\right)\right] G_{w}^{\mathrm{II}}\left(r_{1}, \theta_{1} ; r_{01}, 0\right) d r_{01} \\
& -\int_{R_{1}}^{R_{1}+A_{1}} \tau_{\theta z}^{\mathrm{I}}\left(r_{01}, \pi\right) G_{w}^{\mathrm{I}}\left(r_{1}, \theta_{1} ; r_{01}, \pi\right) d r_{01}+\int_{R_{2}}^{R_{2}+A_{2}} \tau_{\theta z}^{\mathrm{I}}\left(r_{02}, 0\right) G_{w}^{\mathrm{I}}\left(r_{2}, \theta_{2} ; r_{02}, 0\right) d r_{02} \\
& -\int_{R_{1}}^{R_{1}+A_{1}} \tau_{\theta z}^{\mathrm{II}}\left(r_{01}, \pi\right) G_{w}^{\mathrm{II}}\left(r_{1}, \theta_{1} ; r_{01}, \pi\right) d r_{01}+\int_{R_{2}}^{R_{2}+A_{2}} \tau_{\theta z}^{\mathrm{II}}\left(r_{02}, 0\right) G_{w}^{\mathrm{II}}\left(r_{2}, \theta_{2} ; r_{02}, 0\right) d r_{02}
\end{aligned}
$$

The dimensionless DSIFs at the tips of the radial cracks can be defined as

$$
k_{3}^{\sigma}=\frac{1}{\tau_{0} Q}\left|\lim _{r_{0 j} \rightarrow R_{j}+A_{j}} f_{1}\left(r_{0 j}, \theta_{0 j}\right) \sqrt{2\left(r_{0 j}-R_{j}-A_{j}\right)}\right|, Q=\sqrt{R_{j}+A_{j} / 2}, \tau_{0}=-i k_{\mathrm{II}}\left(c_{44}^{\mathrm{II}} w_{2}+e_{15}^{\mathrm{II}} \phi_{2}\right) .
$$

\section{Results and discussions}

In this section, some results for the DSIFs at the right crack's tip are provided to show the influence of the piezoelectric constants, structural geometry, and the frequencies of the incident wave on the DSIFs. It is assumed that two circular cavities have the same radius $R$, and the lengths of the cracks emanating from the edges of the circular cavities are also equal to $A$.

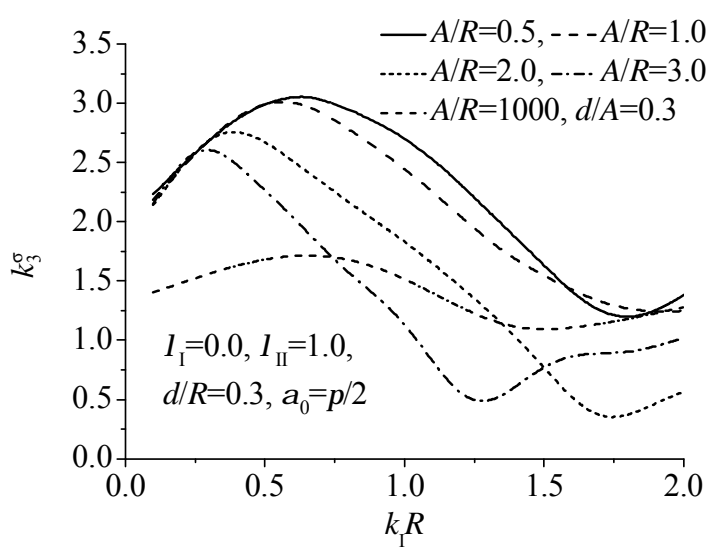

Fig. 3 Variations of DSIFs with $k_{1} R$ for different $A / R$

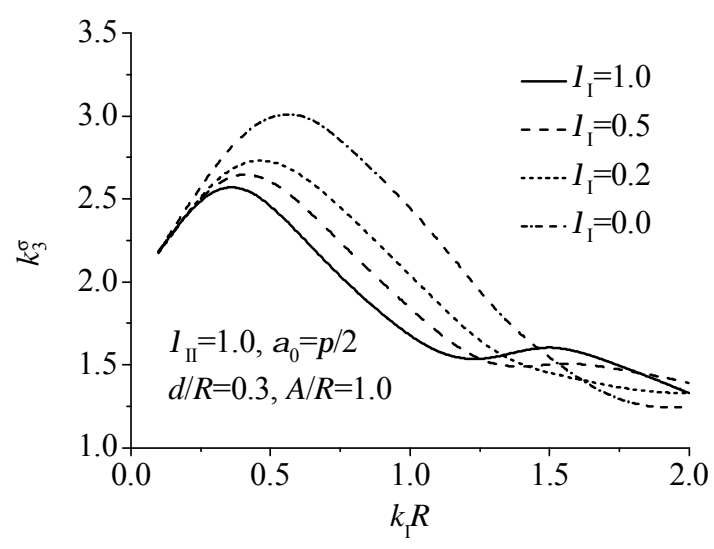

Fig. 4 Variations of DSIFs with $k_{\mathrm{I}} R$ for different $\lambda_{\mathrm{I}}$

Fig. 3 shows the variations of the DSIFs with $A / R$ and $k_{\mathrm{I}} R$. When $A / R=1000$ and $d / A=0.3$, the radial cracks model is reduced to the straight-line crack model. It is compared with the corresponding result in the document [2], it can be seen that they coincide well. The other curves show that DSIFs are larger at lower frequencies $\left(k_{\mathrm{I}} R<1.0\right)$ than those at higher frequencies $\left(k_{\mathrm{I}} R>1.0\right)$. At Fig. 4 , the variations of the DSIFs with $\lambda_{\mathrm{I}}$ and $k_{\mathrm{I}} R$ are shown. It is displayed that the values of DSIFs increase with the decrease of $\lambda_{\mathrm{I}}$ at lower frequencies, which indicates that the mismatch of the materials will increase the stress concentration at the crack's tip. Fig. 5 shows the variations of the DSIFs with $k_{1} R$ and $A / R$. It can be 
seen that the largest values of DSIFs are obtained when $A / R<1$. The values of the DSIFs decrease with the increase of $A / R$. Fig. 6 shows the variations of the DSIFs with $d / R$ and $k_{1} R$. It is displayed that the values of the DSIFs decrease with the increase of the relative distance $d / R$ when $k_{1} R<1.2$, but there is a reverse trend when $k_{1} R>1.2$.

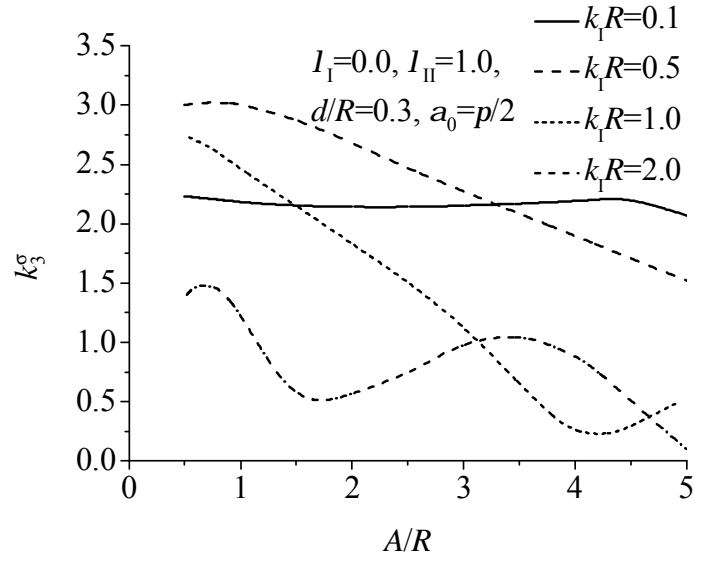

Fig. 5 Variations of DSIFs with $A / R$ for different $k_{1} R$

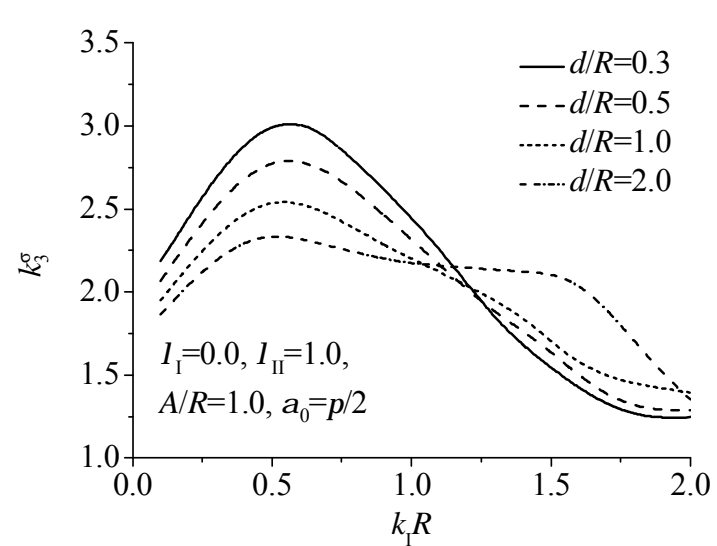

Fig. 6 Variations of DSIFs with $k_{1} R$ for different $d / R$

\section{Conclusions}

The major task of this work is to find the influence of the piezoelectric constants, structural geometry, and the wave frequencies of incident wave upon the local stress field of the cracks' tips. According to parametric analysis, some conclusions can be drawn as follows:

1. Dynamic analyses on piezoelectric bimaterials are more important than those on homogenous piezoelectric medium, because the former may have larger dynamic stress intensity factors.

2. The influence of the cavities on DSIFs is more significant when the crack's lengths and the radii of the cavities have the same order especially in low-frequency situation.

3. The larger values of the DSIFs can be obtained at lower frequencies rather than higher frequencies.

\section{Acknowledgements}

The present work is supported by the National Science Fund for Young Scholars of Hebei Education Department (Q2012031).

\section{References}

[1] F. Narita, Y. Shindo, Theoretical and applied fracture mechanics. 29 (1998) 169-180.

[2] X.D. Wang, International Journal of Solids and Structures. 38 (2001) 815-831.

[3] X.C. Zhong, X.F. Li, Mechanics Research Communications. 32 (2005) 401-410.

[4] Z.G. Zhou, B. Wang, Applied Mathematics and Mechanics. 28(4) (2007) 417-428.

[5] Y.J. Wang, C.F. Gao, International Journal of Solids and Structures. 45 (2008) 4590-4599.

[6] J.H. Guo, Z.X. Lu, H.T. Han, Z. Y. Yang, International Journal of Solids and Structures. 46 (2009) 3799-3809. 